\title{
Situational analysis of mental health system in the West region of Cameroon using the world health organization's assessment instrument for mental health systems (WHO- AIMS)
}

Michael guy TOGUEM, University of Nairobi, department of psychiatry, master's student in psychiatry, Nairobi, Kenya, tgmc91@gmail.com, corresponding author

Manassi KUMAR, University of Nairobi, department of psychiatry, senior lecturer, clinical psychologist, Nairobi, Kenya, $\underline{\text { m.kumar@ucl.ac.uk }}$

David NDETEI, University of Nairobi, department of psychiatry, professor of psychiatry, Nairobi, Kenya,dmndetei@amhf.or.ke

Francois Erero NJENGOUE, University of Douala, department of social sciences, senior lecturer, clinical psychologist, Douala, Cameroon, merengwe@gmail.com

Frederick OWITI, University of Nairobi, department of psychiatry, senior lecturer, psychiatrist, Nairobi, Kenya, f.owiti89@gmail.com 


\begin{abstract}
Background: The burden of mental illnesses is increasing in Cameroon and there is no available published work on the mechanisms put in place to address this issue. The government recognizes this burden and tries to feel the gap. In line to this, this study aimed at describing the mental health services available in West Cameroon to provide an evidence based support to this process.

Method: We used the world health organization assessment instrument for mental health systems (WHO-AIMS) version 2.2 to collect, analyze, and report, data on mental health services offered in 2020 in the west region of Cameroon. We extracted our data from the registers of the different mental health facilities of the region and we interviewed staffs in these facilities and at the ministry of public health.

Results: The region is divided into 20 health districts, of which 06 offered mental health services. As a whole, Cameroon had a mental health policy, mental health plan, but no mental health legislation and emergency plan. There was no specific budget for mental health in the country. In the west region of Cameroon, there was no psychiatrist. Mental health services were offered by nurses, psychologists, general practitioner and neurologists; representing 1.87 human resources in mental health facilities per 100,000 population, of which 1.4 were nurses. 1 in 1.4 of these nurses worked in the main city. There was no formal link of mental health services with other sectors and no publication in the previous 5 years about mental health in the region referenced on PubMed.
\end{abstract}

Conclusion: In 2020, access too mental health services in the west region of Cameroon was unequitable, and was not supported by scientific evidence.

Keywords: Cameroon, mental health system, WHO-AIMS, West region 


\section{Introduction}

Cameroon is a lower-middle-income country (LMIC) in Central Africa with a population of around 25 million. It has two national languages, English and French(1).The life expectancy at birth was 59 years in 2019(2). In Cameroon, neuropsychiatric disorders represent $6.1 \%$ of the burden of all diseases $(3,4)$. Even though the burden of major depressive disorders has been increasing between 1990 and 2010, general practitioners of the city of Douala, Cameroon, diagnose $1.92 \%$ of the cases of depression presenting to their clinics $(5,6)$. In Guidiguis health district in the fare north region, where patients are mainly seen by nurses, $13.33 \%$ of them were able to identify 3 symptoms of depression and knew that it can lead to suicide(7). In the Fako division in South West region, $45.1 \%$ of primary health care providers were not aware if there were any psychotropic drugs available in their area and also $49.1 \%$ did not receive a formal teaching in mental health (4). In the west region, we could not find any information about mental health. The west region is neighbor to the Nord west region which is going through the Anglophone crisis(8). This stressed the need for mental health services in this region where many people are seeking refuge.

The WHO Cameroon mental health state profile published in 2017 don't provide any information on mental health facilities, and human resources available in the country(9). All these, raises concerns about how do Cameroonian individuals with mental illness in the west region find support and care; and what sort of mechanisms are in place for the promotion, restoration or maintenance of mental health. Before we can build a good mental health system, we need to be well informed on the real facts about mental health in the region. This is the reason why our aim in this study is to collect the relevant information in the west region of Cameroon that can be used to make informed decisions geared towards building an efficient mental health system for the region.

\section{Method}

The study was conducted in the West region of Cameroon. It had an estimated population of 1921590 people in 2015, divided into 20 health districts. It has an approximate geographical area of 13,872 square kilometers and a population of $1,921,590$. The proportion of population under the age of 15 years was $44.71 \%(11,12)$..The main language used in the region is French, and the main ethnic group is "Bamileke". Religious groups include Muslim, Animist, Catholics and other

Christian confessions. The population is a mixed of rural and urban population. Data was collected in February 2021 and was based on the year 2020. 
We got the list of the hospitals of the region from the West region's delegation for the ministry of health. Then, we went to all the hospitals of the region and we selected those offering mental health services during the year 2020 to include in our study. While collecting data, in each facility, we asked to the mental health workers we met if they knew any other mental health facility in their health district, if yes, we went to this facility checked if they were offering mental health services in the year 2020. If yes, this other facility was included in the study. We used the World Health Organization Assessment Instrument For Mental Health Systems (WHO-AIMS) version 2.2 to collect, analyze and report data on the mental health system of the west region of Cameroon. We extracted our data from the registers of the mental health facilities of the region, we interviewed the mental health staff of these facilities and we interviewed a key member of mental health at the ministry of public health.

The WHO-AIMS is a tool built by WHO to allow a comparable evaluation of mental health systems using key indicators. This is aimed at providing information for a more efficient and effective building of the mental health system. So far, it has been proven effective in the straightening of the mental health system of several countries including Ethiopia and Morocco. The questions are divided into 6 domains, 28 facets, and 156 items, which are all interdependent. These domains are:

- Domain 1: Policy and Legislative framework

- Domain 2: Mental health services

- Domain 3: Mental health in primary care

- Domain 4: Human Resources

- Domain 5: Public education and link s with other sectors

- Domain 6: Monitoring and research(10).

This is be the first study in the country using this tool.

The data collected was used to answer each question of the WHO-AIMS and are reported in the following section.

\section{Results}

\section{Policy and legislative framework}

Cameroon's mental health policy was last revised in 2016 and includes the following components: (1) developing a mental health component in primary health care, (2) human resources, (3) involvement of users and families, (4) advocacy and promotion, (5) human rights protection of users, (6) equity and access to mental health services across different groups, (7) 
quality improvement, (8) monitoring system, (9) developing community mental health services, and (10) financing. It does not include the downsizing of large mental hospital.

The national essential medicines list includes all the categories of psychotropics: (1) antipsychotics, (2) anxiolytics, (3) antidepressants, (4) mood stabilizers, and (5) antiepileptic drugs

The last revision of the mental health plan took place in 2016 and included: (1) human resources, (2) advocacy and promotion, (3) human right protection of users, (4) financing, and (5) monitoring system. This mental health plan does not include the development of community mental health services, the downsizing of large mental hospitals, any reforming of the mental health hospitals to provide comprehensive care, equity of access to mental health services across different groups and quality improvement. Even though users and families are mentioned in this plan, it is not clear which role they are going to play. A timeframe and specific goals are mentioned in the plans, some of which have been reached in the last year but there is no budget dedicated to mental health. There is no disaster/emergency preparedness plan for mental health and there is no current mental health legislation even though, there are some consideration about mental health in the penal, civic and family code, for example: article 87 of the penal code.

During the year 2020, there was not any inspection or training of mental health care workers on human rights.

There is no specific budget for mental health in Cameroon. All the mental health hospitals of the region are privately own and do not receive money from the government. There is no social assurance scheme and there is no free access to essential psychotropic medicines. One-day treatment of antipsychotic medication is $8.7 \%$ and antidepressant medication is $20.4 \%$ of the minimum daily wage (these calculations are based on the current minimum monthly wage of 36,270 FCFA). Worker's insurance benefits exist but the number of people covered is unknown.

\section{Mental health services}

There is a national mental health authority: vice director of mental health at the ministry of public health. Her office and she (1) provide advice to the government on mental health policy and legislation, (2) they are involved in service, (3) service management but there is no monitoring nor quality assessment of mental health services. In the west region, all community based psychiatric in-patient units are affiliated to a mental health outpatient facility in the same hospital. Both were ran by the same personnel except in the referral hospital of the region. Mental health services are organized in terms of service areas: district. There are 20 districts in the region but only 06 of them have a mental health facility: Batcham, Foumban, Mbouda, 
Dschang, Mifi, and Bangangte. Those not having any often send their mentally sick patients to the closest mental health service.

In the region, there are 08 outpatient mental health facilities and 02 neuropsychiatric unit that offer outpatient services. The neuropsychiatric unit is a combination of a psychiatric unit and a neurological unit. A neurologist who diagnoses and treats psychiatric patients heads it. For the purpose of this research, we are going to consider the neuropsychiatric unit as a psychiatric unit. None of these services is for children and adolescents only. These facilities treated 127 users per 100,000 general population (2,448 users). Of all users treated in mental health outpatient facilities $51 \%$ are female and $19 \%$ are below 18 years old.

The users treated in outpatient facilities are diagnosed with mental and behavioral disorders due to: psychoactive substance (4\%); schizophrenia, schizotypal and delusional disorder (14\%); mood disorders (22\%); neurotic, stress-related and somatoform disorders (14\%); disorder of adult personality and behavior (1\%) and other diagnoses ( epilepsy, organic mental disorders, mental retardation, behavioral and emotional disorder with onset usually occurring in childhood and adolescence, disorders of psychological development) represented (37\%). These other diagnoses were dominated by epilepsy. The total is not adding up to $100 \%$ because of missing information in the registers. The average number of contacts per user is 2 . Half of outpatient facilities provide follow-up care in the community, while 10\% (01) have mental health mobile teams. There is no day treatment facility. In terms of available treatments, $20 \%$ of the outpatient facilities offer psychosocial treatments. Clinical psychologists deliver these psychosocial treatments. $42.9 \%$ of mental health outpatient facilities have at last one psychotropic medicine of each therapeutic class (anti-psychotic, antidepressant, mood stabilizer, anxiolytic, and antiepileptic medicines) available in the facility and all of them have them in the facility or in a near-by pharmacy all year round.

There are 07 community-based psychiatric inpatient units (this includes 02 community-based neuropsychiatric inpatient units) available in the region for a total of 0.62 beds per 100,000 population; this does not include the beds of neuropsychiatric units. None of the beds in community-based inpatient units are reserved for children and adolescents only. Based on the estimations of mental health nurses, one unit had $20 \%$ and another one did not have admitted patient aged below 18 years old. One third of community-based psychiatric inpatient units have at least one psychotropic medicine of each therapeutic class (anti-psychotic, antidepressant, mood stabilizer, anxiolytic, and antiepileptic medicines) available in the facility. There is no community residential facility in the region. 
There are 02 mental hospitals available in the region for a total of 1.6 beds per 100,000 population. All of these facilities are organizationally integrated with mental health outpatient facilities. None of these beds in mental hospitals were reserved for children and adolescents only. Of the users of mental hospitals $40.6 \%$ were female. The diagnoses among all users of mental hospitals were as follows: mental and behavioral disorders due to psychoactive substance use (23.8\%); schizophrenia, schizotypal and delusional disorders (38.1\%); mood disorders (8.8\%); neurotic, stress-related and somatoform disorders (5\%); disorder of adult personality and behavior $(0.6 \%)$ and other $(6.9 \%)$. The number of users of mental hospitals was 8.3 per 100,000 population (160 users). All mental hospitals had at least one psychotropic medicine of each therapeutic class (anti-psychotic, antidepressant, mood stabilizer, anxiolytic, and antiepileptic medicines) available in the facility. There were no forensic nor residential facility in the region.

The status of voluntary/involuntary admission to mental health institutions was not recorded. Nurses reported that, $19 \%$ of patients were restrained or secluded at least once within the last year in community-based psychiatric inpatient units, in comparison to an estimated $30 \%$ of patients in mental hospitals.

The ratio of beds located in Bafoussam which is the largest city of the region compared to those available in the whole region was 3 . Inequity access to mental health services for other minority users (e.g., linguistic, ethnic, religious minorities) is a mild issue in the region.

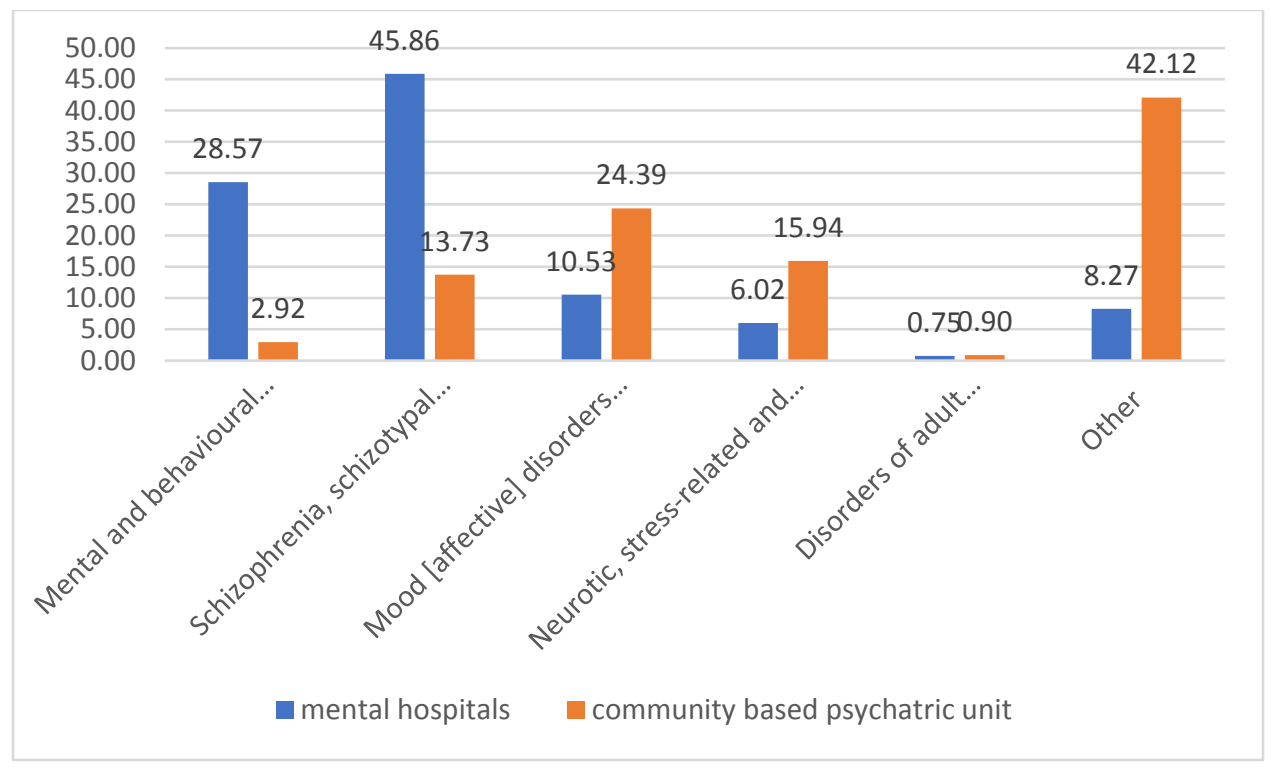

Figure: Percentage of patients treated in mental health facilities by diagnosis 
The predominant diagnosis category on this diagram is schizophrenia, schizotypal and delusional disorders for mental hospitals and other for community based psychiatric units.

\section{Mental health in primary health care}

Based on the harmonized programme for schools of medicine in Cameroon, in the training hours for medical doctors, 0.9 percent is devoted to mental health and this training is only theoretical. Nurses also receive trainings on mental health but we could not get more on this. In terms of refresher training, 02 nurses, belonging to a faith-based hospital, had received at least two days of refresher training in mental health, while none of the primary health care doctors received such a training.

In terms of physician-based primary health care clinics offering mental health

services, few (14.3\%) had assessment and treatment protocols for key mental health conditions available; $50 \%$ of full time primary health care doctors make at least one referral per month to a mental health professional; as a follow up on their transferred patients, $29 \%$ of the primary health care doctors in these hospitals interact at least once a month with the mental health professional and $14 \%$ of these facilities, none of the mental hospitals, interacted with a complementary/ alternative/ traditional practitioner.

Non doctor/non nurse primary health care workers were not allowed to prescribe psychotropic medications in any circumstance. Primary health care doctor and mental health nurses were allowed to prescribe psychotropic medications without restrictions. However, on the ground, this is done by psychiatric nurses and neurologists. As for availability of psychotropic medicines, almost all of physician-based PHC clinics had at least one psychotropic medicine of each therapeutic category (anti-psychotic, antidepressant, mood stabilizer, anxiolytic, and antiepileptic).

\section{Human resources}

The total number of human resources working in mental health facilities per 100,000 population is 1.87 . The breakdown according to profession is as follows: zero psychiatrist, 0.1 neurologist, 0.05 general practitioner, 1.4 nurses, 0.1 psychologists, zero social workers, zero occupational therapists, and 0.2 other health or mental health workers (including auxiliary staff, non-doctor/non-physician primary health care workers, health assistants, medical assistants, professional and paraprofessional psychosocial counselors). Fifty two percent of psychologists and nurses work only for government administered mental health facilities, $45 \%$ only for mental health non-governmental organization / for profit mental 
health facilities/ private practice, and $3 \%$ work for both. One nurse in 1.4 works in the largest city: Bafoussam.

Regarding the workplace, as for the other medical doctors (i.e., those not specialized in mental health), 2 (neurologists) work in mental health outpatient facilities and in the community-based psychiatric inpatient units of the same hospitals, 1 (general practitioner) works only in the community-based psychiatric inpatient unit, and none in mental hospitals. There are 17 nurses working in mental health outpatient facilities, 16 in community-based psychiatric inpatient units and 10 in mental hospitals. As for the other mental health professionals, there are 02 psychologists working in hospitals that include an outpatient facility and a community-based psychiatric inpatient unit; each of them covers both units. None of them works in a mental hospital. Finally, regarding the other health or mental health workers, 04 work in mental hospitals, while none are working in community-based psychiatric inpatient units and outpatient facilities because in these facilities, there belong to other units and assist in case they are needed.

In terms of staffing in mental health facilities, there are 1.3 nurses per bed assigned specifically for psychiatric patients in community-based psychiatric inpatient units, compared to 0.33 per bed in mental hospitals. Finally, for other mental health care staff (e.g., psychologists, social workers, occupational therapists, other health or mental health workers), there are 0.17 per bed for community-based psychiatric inpatient units while there is none in mental hospitals. About the distribution of human resources for mental health between urban and rural areas, one nurse in 1.4 works in the largest city: Bafoussam.

In the region, one school trains mental health nurse but no graduation so far. There is only one school of medicine and several schools of nursing, these other schools do not train mental health professionals. Among the mental health care staff, one nurse attended refresher training on the rational use of drugs while none of them attended a refresher training on psychosocial interventions, or child/adolescent mental health issues. There are no consumer or family associations in the region.

\section{Public education and links with other sectors}

There is a foundation in the region: Fondation Olympia Jujitsu Cameroun (FOJCAM) that oversees public education and awareness campaigns on psychoactive substance use in the whole country. These campaigns have targeted the following groups: general population, adolescents, and women. There haven't been any public educational awareness campaigns targeting professional groups. 
Now, there is no legislative or financial support for employment, provision against discrimination at work and discrimination in housing for people with mental disorders. There is no formal collaborative program with other health and non-health agency.

There is no mental hospital providing employment to people with severe mental disorder. Information on mental health and schools is not available but from what was gathered from the mental health workers of the region, none of them work for a school. Only one nurse provides mental health services in one of the prisons of the region and he sees at least one prisoner per month from the prison of his district. The mental health workers of the region didn't offer any educational activity to police officers, judges, laws. Finally, there is no social welfare benefit for disability in the region.

\section{Monitoring and research}

A formally defined list of individual data items that ought to be collected by all mental health facilities exists and includes the number of inpatient admissions, number of days spent in hospital, diagnosis, and number of users treated even though none of these facilities collected data on the number of days spent in admission. This information is sent

after every trimester to the west regional delegation for the ministry of public health. There is no report produced on mental health services by the government. None of the mental health professionals of the region is involved in research. There hasn't been any publication on mental health in the region in the past five years. In the same time, there are 40 health related publications from the region identified on PubMed.

\section{Discussion}

\section{Policy and legislative framework}

Mental health is one of the priority areas within the Ministry of Health's strategic plan (13). This might explain why we found some success from both the government and the private sector about mental health services in the West region of Cameroon. These services are being provided in physician based primary health care, in the referral hospital of the region and in two mental hospitals. These mental hospitals are staffed with nurses only. Currently, the service covers 6 of the 20 health districts areas of the region. The commitment of the Cameroonian government towards mental health can also be seen in the efforts they put in place to address the mental health aspect of the corona virus disease 19 pandemic (14). 
The last version of the mental health policy and plan was developed in 2016, the minister of public health approved them. The mental health policy addresses all the strategic aspect needed, except downsizing of mental health hospitals. This might be because currently in Cameroon, the public mental hospitals available are all integrated within general hospitals. Together with the privately own mental hospitals, they are poorly specialized and only offer short-term services. Therefore, there is a need to include in the planning, the construction of comprehensive infrastructures for mental health services, including highly specialized, long stay mental hospital, forensic units, and make these services accessible to the populations of the whole country. There should also be community mental health services and a promotion of informal mental health services (15). The mental health plan failed to take into consideration some key aspects such as equity of access to mental health services, improvement of the package of services, and the development of community mental health services. Some efforts are needed to improve its quality and its impact. These insufficiencies might be due to the failure to include, the patients, their families; representatives from the ministry of finance, ministry of justice, social welfare, and private sector in the development of these documents. In addition, it has been reported that the mental health policy is not available in hard copy. As mentioned by some mental health professionals in the country, their quality is questionable. For example, under mental health financing, the amount and the source of money that needs to be allocated are not mentioned together with the number of human resources needed. These documents are supported by evidence from some few hospital-based studies, which is not enough. And, there are several other quality issues that can be raised (16-19). Some of these findings are not unique to Cameroon. WHO reports that only half of the lower middle income countries with a mental health policy report an estimation of the financial and/or human resources and, in Africa, $72 \%$ of the countries have a standalone mental health policy and/or plan(20). There is a need for the development of mental health legislation and emergency plan. The mental health legislation is indeed very important because people with mental disorders are highly stigmatized and marginalized in Cameroon (19). This might thereby help in promoting their rights and improve their management (21). We also need a mental health emergency plan, particularly because of the current Anglophone crisis in the English speaking regions of the country (22).

One-day treatment of medication is $8.7 \%$ and $20.4 \%$ of the minimum daily wage. This seems better than the $37 \%$ and $7 \%$ observed in Uganda, respectively for antipsychotic and antidepressant medication (23). 


\section{Mental health services}

Currently in Cameroon, there is no monitoring of the mental health services being offered. The mental health department at the Ministry of Health might benefit by putting a monitoring system in place. This might help them to have a better understanding of the process being deployed and build a more efficient approach to address the mental health needs of the population. The most commonly found diagnosis categories in this population was schizophrenia, schizotypal and delusional disorders at $45.86 \%$ in mental hospitals and other diagnosis ( epilepsy, organic mental disorders, mental retardation, behavioral and emotional disorder with onset usually occurring in childhood and adolescence, disorders of psychological development) at $42.12 \%$ in community based psychiatric units. They are several factors which can contribute to this pattern. They were two hospitals with a neuropsychiatric unit, of which one had the highest number of psychiatric patients seen in the year 2020. These neuropsychiatric units are headed by neurologists whom are the ones making diagnosis. These neurologists are likely to attract more of the psychiatric patients with neurological manifestations, and they are likely to attribute a neurological diagnosis to a psychiatric condition thereby inflating the proportion of patients with neurological diagnosis. The high proportion of patients with schizophrenia, schizotypal and delusional disorder in mental hospitals might be explained by the fact that these are usually very severe and chronic conditions, making it more likely to arrive to the hospital. A similar pattern was observed in Ghana where the predominant diagnosis in outpatient mental health services was; other (39\%) followed by schizophrenia (25\%) and the predominance of schizophrenia $(21 \%)$ in community based psychiatric in patient unit, other not taken into consideration. Also schizophrenia was the most common diagnosis in mental hospitals: $32 \%$ (24). These diagnoses reported in the records presented some issues. We observed some diagnosis such as "schizophrenie induite par l'usage de substance psychoactive" (substance induced schizophrenia), "psychose maniaco depressive" (maniaco depressive psychosis), "psychose hallucinatoire chronique" (chronic hallucinatory psychosis) and more which raised some concern about the quality of the diagnosis made because none of these diagnosis exist in the International Classification of Diseases 10 or in the Diagnostic and Statistical Manual of Mental Disorders, 5th Edition (25,26). The number of contact of 2 per patient we found is small compare to the 4.99 found in Ghana(24). We couldn't identify any reason for this loss of follow up. This might be an interesting area of exploration in the future. In community based psychiatric, inpatient units nurses estimated that $19 \%$ of patients were either secluded or physically restrained. This is higher than the $10 \%$ reported in Ghana and lower than the $33 \%$ reported on average in LMICs $(24,27)$. 
The psychiatric beds density in the largest city: Bafoussam is 3 times the beds available in the entire region. This ratio is similar to the average 2.9 found in LMIC (27). It illustrates lower access of rural populations to mental health services.

\section{Mental health in primary health care}

Less than $1 \%$ of the training of medical doctors is devoted to mental health in Cameroon, this is very small compared to the $10 \%$ observed in Uganda, and the average 3\% in LMICs $(23,27)$. In addition, this training in Cameroon does not include any practical exposure to mental health. This might explain the less than $2 \%$ diagnosis rate of depression by general practitioners of Douala, Cameroon (28). To address this, the ministry of higher education and the ministry of public health should develop a comprehensive program to equip doctors and nurses with skills to address mental health issues. Assessment and treatment protocols were only available in few physician based PHC, which is similar to the findings in Uganda and is the general tendency in LMIC $(23,27)$. These protocols should be developed and made available, taking into consideration the recommended drugs and the emergency psychiatric conditions that can be found in Cameroon. There was no interaction with traditional healers, contrary to what was found in Ghana (24). This interaction shall be built because it helps in providing a better care to patients (20).

\section{Human resources}

The average number of human resources working in mental health facilities of 1.87 per 100.000 population is way below the 6 per 100.000 population observed in LMICs, and is above the African average of 1.3 per 100.000 population. Of these 1.87 per 100.000 population, there is no psychiatrist. We should acknowledge the fact that by the time we were ending with our data collection, the first psychiatrist of the west region of Cameroon settled in the referral hospital of the region. The school of psychiatric nursing in the region, the postgraduate program of psychiatry, and psychology that are in the country might further contribute to fill this gap in the future. The amount of people trained needs to be further aligned with an assessment of the needs of the population in terms of mental health.

\section{Public education and links with other sectors}

We could only find one mental health promotion program in the region, designed for psychoactive substance use, where, $52 \%$ of the African countries have less than 2 functional programs. The public education needs also to be planned and executed by the same team that built the mental health plan. This has also to be expanded to other areas such as suicide prevention, domestic violence, mental health stigma, and more (20). There is 
also a need for formal agreement of mental health services with other sectors for the mental health service to be able to reach those in prisons, at school, and all the other area where these services are highly needed.

\section{Monitoring and research}

As a starting point for an efficient and effective mental health system in West Cameroon, the environment needs to be enable to facilitate research which will serve to build an evidence based mental health service in the region.

\section{Conclusion}

The current mental health system in west Cameroon still have a lot to improve on. However, some significant effort from the government to fill this gap can be noticed. One mental health nursing school created in the region in the past 2 years, services covering 6 out of the 20 districts, the first psychiatrist in the region arriving during our data collection. This effort needs to use an evidence based, and comprehensive approach, with a big focus on quality. The main recommendations we can make are that the government should:

- Maintain the good things that have been started already

- A more comprehensive approach should be used. That is, including research, systemic planning, training, service delivery, monitoring system

- More studies need to be done to have a good understanding of the need in terms of mental health services in the region and the decisions should reflect on these studies

- The process to address mental health issues should include all the protagonists of mental health. That is: the private sector, the faith organizations, patients and their families, and so on.

- There should be a focus on quality

- A monitoring system should be put in place to assess the evolution of the process and make corrections when needed.

\section{Limitations}

The information collected in this study was gotten from key informants and records. The information gotten form key informants may be reflecting their opinion more than the actual fact. The information gotten form records were altered by some missing information and the quality of the information reported. Some indicators such as the number of psychiatric beds available in the community based psychiatric inpatient unit may not really 
reflect the size of the service offered because most of the time even though they didn't have exclusive beds, they could hospitalize on any bed available in another unit. Some mental health units frequently have student that are there for internships and thereby contribute to the service being offered but were not captured in our study. Some other personnel were trained in HIV programs to offer psychological support to HIV patients but they were not linked with the formal mental health system of the region and were not captured in the study. These data reflect the year 2020 and things seems to be changing on the ground in Cameroon.

\section{List of abbreviations}

WHO: World Health Organization

WHO-AIMS: World Health Organization Assessment Instrument for Mental Health Systems

LMIC: Lower-Middle-Income Country

\section{Declarations}

Not applicable

\section{Competing interests}

The authors declare that they have no competing interests

\section{Acknowledgements}

We thank the delegate of the ministry of health for the west region of Cameroon; the directors of the hospitals where data was collected for allowing us to collect data in their institutions. We thank the office staff of mental health of the ministry of health and the mental health workers of the west region of Cameroon for providing us with the registers and answering to our questions.

\section{Funding}

The study was entirely funded by the primary investigator.

\section{Authors' contributions}


MT is the primary investigator, he chose the topic, did the literature search, participated in the design of the study, collected data, analyzed the data, and did all the writings. MK initiated this research idea, participated in the design of the study, and reviewed the work at each step. DN participated in the design of the study, and reviewed the work at each step. FN reviewed the work at each step. FO reviewed the work at each step.

\section{References}

1. The World Bank. Cameroon Overview [Internet]. 2020 [cited 2020 Mar 20]. Available from: https://www.worldbank.org/en/country/cameroon/overview

2. The World Bank. Life expectancy at birth, total (years) - Cameroon |Data [Internet]. 2021 [cited 2021 Jun 22]. Available from: https://data.worldbank.org/indicator/SP.DYN.LE00.IN?locations=CM

3. commonwealth. Mental health in Cameroon [Internet]. 2020 [cited 2020 Mar 19]. Available from:

http://www.commonwealthhealth.org/africa/cameroon/mental_health_in_cameroon/

4. Mulango ID, Atashili J, Gaynes BN, Njim T. Knowledge, attitudes and practices regarding depression among primary health care providers in Fako division, Cameroon. BMC Psychiatry. 2018;18(1):1-9.

5. Institute for Health Metrics and Evaluation. GLOBAL BURDEN OF DISEASES, INJURIES, AND RISK FACTORS STUDY 2010 [Internet]. 2010 [cited 2020 Mar 21]. Available from: http://www.healthmetricsandevaluation.org

6. Toguem MG, Christian E, Fotso JBD. Attitude of general practitioners towards depressive disorders in the city of douala in cameroon. Pan Afr Med J [Internet]. 2019 [cited 2020 Dec 25];34. Available from: https://pubmed.ncbi.nlm.nih.gov/31803341/

7. Keugoung B, Kongnyu ET, Meli J, Criel B. Profile of suicide in rural Cameroon: are health systems doing enough? Trop Med Int Heal [Internet]. 2013 Aug 1 [cited 2020 Mar 19];18(8):985-92. Available from: http://doi.wiley.com/10.1111/tmi.12140

8. Lassaad BA. Cameroun - Crise anglophone : Les principales dates d'une insurrection (Chronologie) [Internet]. 2017 [cited 2021 May 25]. Available from:

https://www.aa.com.tr/fr/afrique/cameroun-crise-anglophone-les-principales-dates-d-uneinsurrection-chronologie/992032

9. WHO. Mental Health ATLAS 2017 Member State Profile. 2018 [cited 2020 Mar 29];1. Available from: https://www.who.int/mental_health/evidence/atlas/profiles2017/CMR.pdf?ua=1 
10. WHO. World Health Organization Assessment Instrument for Mental Health SystemWHO. (2005). World Health Organization Assessment Instrument for Mental Health Systems WHO-AIMS.s WHO-AIMS. 2005.

11. BUCREP BC des R et des E de P. Troisième Recensement Général de la Population et de l’Habitat. 2015;III:91. Available from: http://www.bucrep.cm/index.php/fr/home/137josetta-2-fr-fr/activites-fr-fr-2/projets-en-cours-fr-fr-2/311-enquetes-et-etudes-en-matierede-population-et-developpement

12. CVUC. Région de l'Ouest [Internet]. 2014 [cited 2021 Apr 8]. Available from: http://www.cvuc-uccc.com/national/index.php/fr/carte-communale/region-de-louest

13. MoH. National health development plan. 2016;142. Available from: https://www.minsante.cm/site/?q=en/content/national-health-development-plan-nhdp2016-2020

14. Mviena JLM, Fanne M, Gondo R, Mwamelo AJ, Esso L, Epée E, et al. How mental health care is changing in Cameroon because of the COVID-19 pandemic [Internet]. Vol. 7, The Lancet Psychiatry. Elsevier Ltd; 2020 [cited 2021 Apr 21]. p. e62-3. Available from: https://www.

15. WHO. The optimal mix of services for mental health. 2003. p. 6.

16. MoH. Politique nationale de sante mentale. 2016;26.

17. WHO. Checklist for evaluating a mental health policy [Internet]. [cited 2021 May 2]. Available from: https://www.who.int/mental_health/policy/WHOPolicyChecklist_forwebsite.pdf?ua=1

18. WHO. checklist for evaluating a mental health plan [Internet]. [cited 2021 May 2]. Available from: https://www.who.int/mental_health/policy/WHO PlanChecklist for Website_BlueFormatted.pdf

19. MoH. Programme national de sante mentale. 2016;

20. WHO. Mental health atlas 2017. 2018.

21. WHO. Mental Health Policy and Service Guidance Package [Internet]. 2003 [cited 2021 May 3]. Available from: https://www.who.int/mental_health/policy/services/7_legislation HR_WEB_07.pdf

22. O’Grady S. Cameroon Anglophone crisis: A crackdown on English speakers is fueling support for Ambazonia - Washington Post [Internet]. 2019 [cited 2021 May 3]. Available from: https://www.washingtonpost.com/graphics/2019/world/cameroon-anglophonecrisis/

23. Kigozi F, Ssebunnya J, Kizza D, Cooper S, Ndyanabangi S. An overview of Uganda's mental health care system: Results from an assessment using the world health 
organization's assessment instrument for mental health systems (WHO-AIMS). Int J Ment Health Syst [Internet]. 2010 Jan 20 [cited 2020 Jul 31];4(1):1. Available from:

http://ijmhs.biomedcentral.com/articles/10.1186/1752-4458-4-1

24. Roberts M, Mogan C, Asare JB. An overview of Ghana's mental health system: results from an assessment using the World Health Organization's Assessment Instrument for Mental Health Systems (WHO-AIMS). Int J Ment Health Syst [Internet]. 2014 May 4 [cited $2020 \mathrm{Jul} \mathrm{31];8(1):16.} \mathrm{Available} \mathrm{from:}$ http://www.ncbi.nlm.nih.gov/pubmed/24817908

25. APA. DIAGNOSTIC AND STATISTICAL MANUAL OF MENTAL DISORDERS. 5th ed. Washington, London: American psychiatric publishing; 2013. 947 p.

26. WHO. ICD-10 Version:2016 [Internet]. 2016 [cited 2021 May 3]. Available from: https://icd.who.int/browse10/2016/en

27. WHO. Mental health systems in selected low- and middle-income countries: a WHOAIMS cross-national analysis. 2009;108. Available from: https://www.who.int/mental_health/evidence/who_aims_report_final.pdf

28. Toguem MG, Christian E, Fotso JBD. Attitude of general practitioners towards depressive disorders in the city of douala in cameroon. Pan Afr Med J. 2019;34. 